\title{
Geospatial Disaster Response during the Haiti Earthquake: A Case Study Spanning Airborne Deployment, Data Collection, Transfer, Processing, and Dissemination
}

\author{
Jan A.N. van Aardt, Donald McKeown, Jason Faulring, Nina Raqueño, May Casterline, \\ Chris Renschler, Ronald Eguchi, David Messinger, Robert Krzaczek, Steve Cavillia, \\ John Antalovich, Jr., Nat Philips, Brent Bartlett, Carl Salvaggio, Erin Ontiveros, and Stuart Gill
}

\begin{abstract}
Immediately following the 12 January 2010 earthquake in Haiti, a disaster response team from Rochester Institute of Technology, ImageCat Inc., and Kucera International, funded by the Global Facility for Disaster Reduction and Recovery group of the World Bank, collected $0.15 \mathrm{~m}$ airborne imagery and two points $/ \mathrm{m}^{2}$ lidar data for $650 \mathrm{~km}^{2}$ over a period of seven days. Data were transferred to Rochester, New York for processing at rates that approached $400 \mathrm{Mb} / \mathrm{s}$ using Internet2, ortho-rectified with a 24-hour turnaround, and distributed to response agencies through file or disk transfer. A unique response effort, dubbed the Global Earth Observation - Catastrophe Assessment Network (GEO-CAN) and headed by ImageCat, utilized over 600 experts from 23 different countries to generate rapid turnaround damage assessment products. This paper highlights the airborne data collection, transfer, processing, and product development effort, which arguably has raised the bar in terms of response to large-scale disasters.
\end{abstract}

\section{Introduction}

On 12 January 2010, a magnitude $7 \mathrm{M}_{\mathrm{W}}$ earthquake struck the nation of Haiti and resulted in over 200,000 people killed and over one million displaced people (World Bank, 2010). The need for accurate high-resolution imagery, detailed surface elevation, and various information maps became evident as the disaster unfolded. Specific remote sensing

Jan van Aardt, Donald McKeown, Jason Faulring, Nina Raqueño, May Casterline, David Messinger, Robert Krzaczek, Steve Cavillia, Brent Bartlett, Carl Salvaggio, and Erin Ontiveros are with the Rochester Institute of Technology, Chester F. Carlson Center for Imaging Science, 54 Lomb Memorial Drive, Rochester, NY, 14623 (vanaardt@cis.rit.edu).

Chris Renschler is with the Department of Geography, 105 Wilkeson Quad, University at Buffalo, Buffalo, NY, 14201.

Ronald Eguchi is with ImageCat, Inc., 400 Oceangate, Suite 1050; Long Beach, CA, 90802.

John Antalovich, Jr. and Nat Philips are with Kucera International, 38133 Western Parkway, Willoughby $\mathrm{OH}, 44094$.

Stuart Gill is with the World Bank, 1818 H Street, N.W., Washington, D.C. 20433. product requirements included building damage assessment (World Bank), debris location and quantification, hydrological mapping (relief agencies), and assessment of the fault line (United States Geological Survey (USGS)). The geospatial community responded by mobilizing various air- and spaceborne remote sensing resources, e.g., the National Oceanic and Atmospheric Administration (NOAA), Google, Inc., Pictometry International, DigitalGlobe, and GeoEye, Inc. were among the responders that started to acquire especially high spatial resolution multispectral imagery over the impacted areas. The Digital Imaging and Remote Sensing Laboratory (DIRS), in the Chester F. Carlson Center for Imaging Science at the Rochester Institute of Technology (RIT) was contracted by the World Bank, through commercial partner ImageCat, to mobilize an airborne imaging system for a large area mapping mission. The Wildfire Airborne Sensor Program (WASP) sensor, developed under funding from the National Aeronautics and Space Administration (NASA) to support wildfire detection and mapping (McKeown et al., 2004; WASP, 2011) and a light detection and ranging (lidar) system were flown over the devastated area from 21-27 January 2010. The resultant $650 \mathrm{~km}^{2}$ multimodal dataset consists of more than 15,000 image frames in each of the visible, shortwaveinfrared (SWIR), midwave-infrared (MWIR), and longwaveinfrared (LWIR) spectral domains, as well as 2.9 billion lidar returns. These public domain data provided a unique capability to characterize the damage and was used for several applications, e.g., building damage assessment, hydrological mapping, and structural assessment of the fault line which has spurred efforts in long-term research and development. The collaborative effort between ImageCat, Inc., RIT, and Kucera International was facilitated by the "Information Products Laboratory for Emergency Response,” a unit dedicated to development of user-driven disaster response products and funded by the National Science Foundation Partnerships for Innovation program. This paper is not intended as a pure scientific description of the effort with associated hypotheses and results, but rather as a high-level description of the efforts that support such data collection and use towards generation of operational disaster response

Photogrammetric Engineering \& Remote Sensing Vol. 77, No. 9, September 2011, pp. 943-952.

0099-1112/11/7709-0943/\$3.00/0

(C) 2011 American Society for Photogrammetry and Remote Sensing 
products. The full effort spanning a system description, deployment, data collection, transfer, processing, and dissemination, to product development will be presented.

\section{Sensor System Overview}

The RIT WASP sensor originally was developed for wildfire mapping research and has been a workhorse system for various phenomenology studies and for investigating the application of remote sensing to emergency response (Li et al., 2005 and 2007; Ononye et al., 2007). The WASP sensor is composed of three Indigo Phoenix infrared imagers in the SWIR (1.0 to $1.7 \mu \mathrm{m})$, MWIR (3.0 to $5.0 \mu \mathrm{m})$, and LWIR (8.0 to $9.2 \mu \mathrm{m})$ spectral regions and a 11 mega-pixel Geospatial Systems, Inc. KCM-11 high-resolution color camera (McKeown et al., 2004; Arsenovic et al., 2009). WASP also incorporates a deployable thermal calibration system that is used to calibrate the MWIR and LWIR cameras to aperture reaching radiance (Arsenovic et al., 2009). Figure 1 shows the configuration of the WASP cameras in a common mounting structure. The figure also shows the in-flight deployable thermal calibration plates that enable accurate estimation of aperture reaching radiance for the MWIR and LWIR cameras. This feature mitigates the large variations in measured focal plane (sensor) radiance caused by thermal emissions from the camera lenses themselves as they change temperature during flight.

The spatial resolution of the color camera is approximately $0.15 \mathrm{~m}$ at $760 \mathrm{~m}(2,500$ feet $)$ above ground level, while the infrared cameras have a spatial resolution of 0.83 $\mathrm{m}$ at this altitude. Each of the four cameras is geometrically calibrated using a three-dimensional calibration cage at RIT that includes both visible and infrared point targets. Figure 2 shows sample imagery that highlights the diversity of information contained in a single WASP image dataset.

The WASP cameras are coupled to an Applanix POS AV310 inertial navigation system (INS) to enable accurate georeferencing of imagery; the inertial measurement unit
(IMU) component of the INS contains precision fiber optic gyros and accelerometers and is mounted to the back of the color camera mounting structure. Typical ground location accuracies from imagery are $<1 \mathrm{~m}$ when using direct georeferencing without ground control points at a low operating altitude.

RIT personnel worked with Kucera International, an aerial mapping company based in Willoughby Ohio, to configure the WASP sensor to interface with the company's single engine Cessna 206 and twin engine Piper Navajo aircraft in support of data collection experiments. The Piper Navajos have a high speed and long range, and Kucera engineered two large viewports, allowing the WASP sensor to be operated simultaneously with other sensors, such as a lidar sensor. Kucera owns and operates a Leica ALS-60 lidar with multiple return measurement capability. Point measurement accuracy is typically $0.15 \mathrm{~m}$ without ground control. The WASP and lidar instruments provide a uniquely comprehensive dataset as a multi-modal sensor suite. Table 1 summarizes the performance parameters of the WASP/lidar sensors.

\section{Deployment and Operations}

Immediately after the earthquake struck in Haiti, RIT began consultation with ImageCat and the World Bank regarding the possibility of deployment of the WASP sensor with a simultaneously operated lidar system to the disaster area. The World Bank team for the Global Facility for Disaster Reduction and Recovery (GFDRR) required high spatial resolution imagery and detailed 3D surface measurements to conduct post-disaster needs assessment (PDNA). ImageCat, a company specializing in global risk and disaster management, was instrumental in coordinating data collection requirements with the World Bank and served as the prime contractor for the RIT operation.

The initial concept was to deploy to the Bahamas as a base of operations for the daily flights over Haiti and then return to Rochester, New York for image processing and dissemination. The mission eventually spanned seven
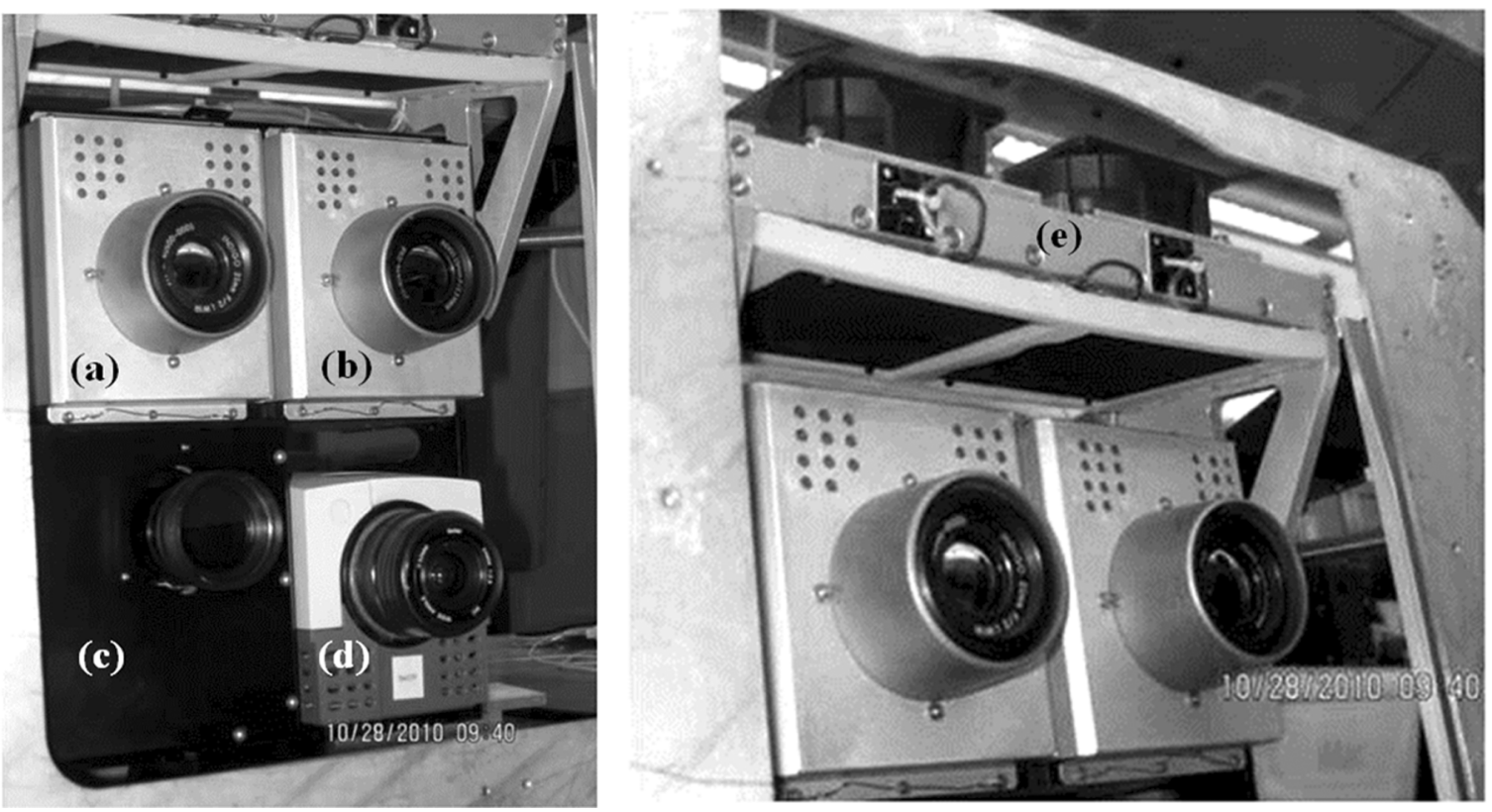

Figure 1. WASP camera and thermal calibration configuration showing the (a) MWIR, (b) LWIR, (c) color, and (d) SWIR cameras, as well as (e) the in-flight deployable calibration plates 


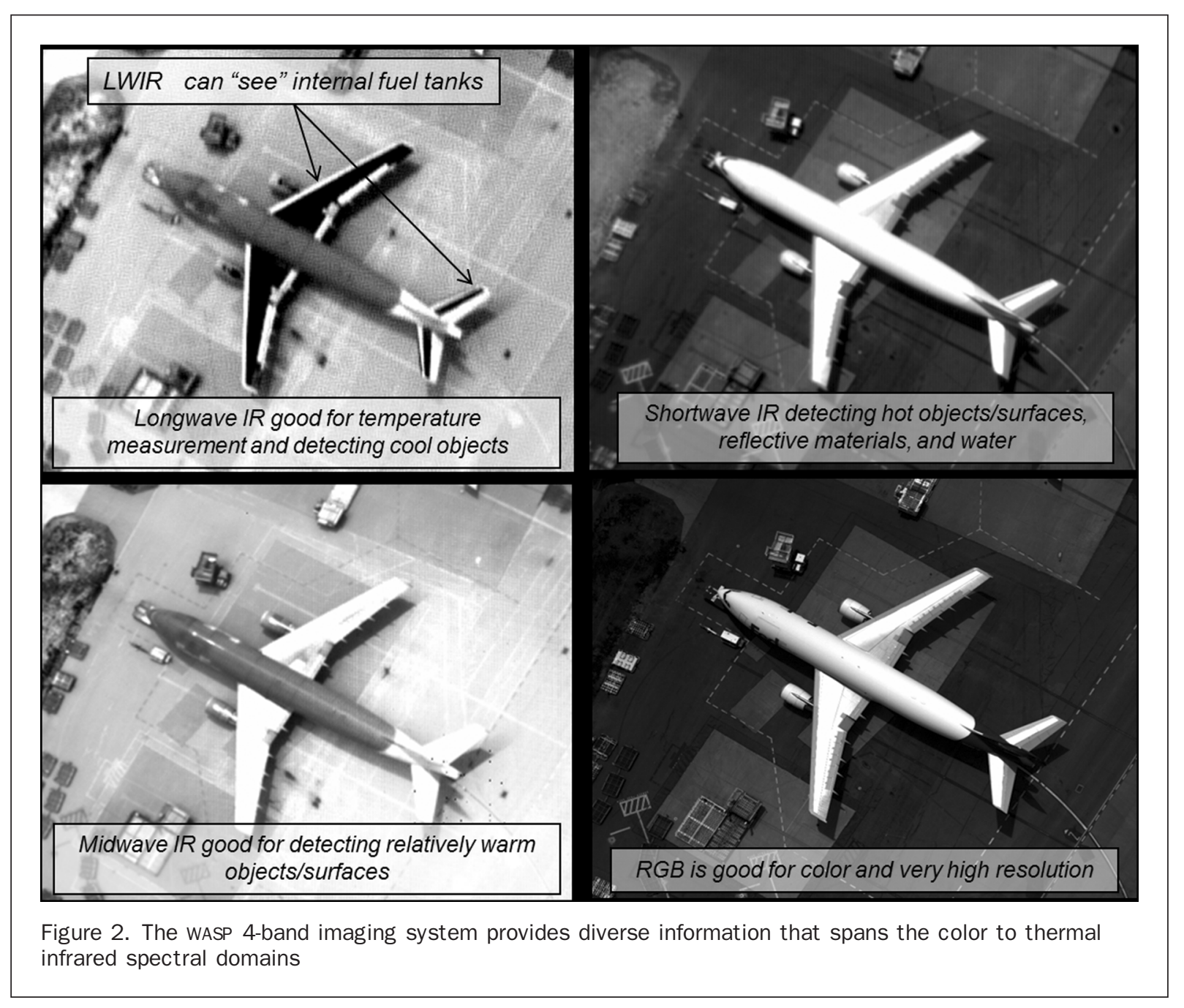

continuous days of flight operations as events unfolded, covering approximately $650 \mathrm{~km}^{2}$ and requiring the transfer of hundreds of gigabytes of imagery data over the Internet from the Caribbean to Rochester for round-the-clock data processing and dissemination.

The team encountered two immediate hurdles as part of our deployment to Haiti. The first was a United States State Department export restriction on our sensor, imposed under International Traffic in Arms Regulations (ITAR), due to the military grade technology contained in the Applanix INS. The professional support from State Department personnel enabled the team to secure an export license on Monday, 18 January 2010. The specifics of the license allowed for operation of the sensor outside of the US, but required that all data transfers from the aircraft take place on US soil. Secondly, the response team needed to coordinate their operations in Haitian airspace. Conditions in Haiti were challenging a week after the earthquake struck and the Haitian air traffic control infrastructure (radar surveillance) was essentially non-existent. In spite of this, there was a heavy flow of relief flights into and out of Port-au-Prince, as well as many low altitude helicopter operations around the area.

Communication with the appropriate air traffic control authority presented a significant challenge. Personnel from NOAA and the Federal Emergency Management Agency (FEMA) were an invaluable resource as they had initiated Haiti operations several days prior to the ImageCat-RIT-World Bank team's arrival and thus were able to provide suggestions in

Table 1. Summary of the Rit wasp-lidar Multi-modal Sensor Suite under the Flight Parameters for the Haiti Response Efforts

\begin{tabular}{|c|c|c|c|c|c|c|}
\hline $\begin{array}{l}\text { Spectral region } \\
\text { (sensor) }\end{array}$ & $\begin{array}{l}\text { Wavelength } \\
\text { (microns) }\end{array}$ & $\begin{array}{l}\text { Spatial } \\
\text { Resolution }(\mathrm{m})^{*}\end{array}$ & Bit Depth & $\begin{array}{l}\text { Temperature } \\
\text { Sensitivity (K) }\end{array}$ & $\begin{array}{l}\text { Geo-location } \\
\text { error }(\mathrm{m})^{* *}\end{array}$ & $\begin{array}{l}\text { Point Density } \\
\left(\mathrm{pts} / \mathrm{m}^{2}\right)\end{array}$ \\
\hline Color & $0.4-0.9$ & 0.15 & 10 & I & $<1$ & I \\
\hline SWIR & $1.0-1.7$ & 0.83 & 14 & I & $<1$ & I \\
\hline MWIR & $3.0-5.0$ & 0.83 & 14 & $<0.1$ & $<1$ & I \\
\hline LWIR & $8.0-9.2$ & 0.83 & 14 & $<0.1$ & $<1$ & l \\
\hline ALS-60 lidar & 1.064 & I & I & I & 0.15 & $2-5$ \\
\hline
\end{tabular}

* at $760 \mathrm{~m}$ altitude

** with differential GPS 
terms of local air traffic liaisons. US Southern Command (SOUTHCOM) provided critical coordination for the operation of US-registered aircraft in the area. The response team provided SOUTHCOM with a summary of the planned operation on a daily basis, plus a pre-briefing for the next day, including the planned coverage area, operating altitudes, and times of flight. SOUTHCOM restricted all local helicopter traffic to a maximum altitude of $150 \mathrm{~m}$ (500 feet) above ground level (AGL) to minimize the chances of separation issues with the team's Navajo flying at $760 \mathrm{~m}$ (2,500 feet) AGL. Finally, there was a daily teleconference hosted by the USGS that included representation from FEMA, the United States Agency for International Development (USAID), National Geospatial Intelligence Agency (NGA), Department of Defense, World Bank, and others to review all planned airborne collection activities by those operating in the area. This initiative enabled valuable communication between parties.

The Kucera Piper Navajo is equipped with extended range Nacelle fuel tanks, which increases the time the aircraft can stay aloft from approximately four to six hours (plus a safety reserve) while flying at a nominal ground speed of 140 knots. However, longer flight durations were offset by the fact that data from the system could only be removed from the airplane on US soil per the export licensing, hence the flight crew operated out of Aguadilla, Puerto Rico for the first four days (21-24 January 2010) of the mission. Refueling stops were made in Puerto Plata, Dominican Republic on the way to and back from Haitian airspace in order to maximize the area collected each day. The flight from Puerto Plata to the affected area in Haiti required approximately an hour, which left four hours of fuel to perform the imaging mission and return to Puerto Plata for refueling.

Upon returning to Aguadilla, Puerto Rico, a local backup copy of the flight data was made and the flight data disks were transported to the University of Puerto Rico at Mayaguez (UPRM) for uploading to Rochester using Internet2. Data were processed at RIT and posted to an FTP server for dissemination over the Internet and in some cases copied to hard drives for shipment. Operations for the last three days (25-27 January 2010) were based from Puerto Plata to expedite observations of the remaining outlying areas. These final acquired data could not be removed from the aircraft, as per the export control license, hence processing activities resumed when the crew returned to Rochester on 28 January 2010. A schematic of the airborne data collection efforts is shown in Plate 1.

As stated above, the effort focused on the Port-au-Prince area during 21-24 January 2010, while severely damaged areas outside the main city were imaged during 25-27 January 2010. Lidar data (2.9 billion returns) were collected at a nominal point density of 2 points $/ \mathrm{m}^{2}$, governed by imagery collection parameters over the inhabited areas of interest, and 5 points $/ \mathrm{m}^{2}$ for high-resolution 3D mapping of the fault line (27 January 2010). Ultimately, over 15,000 frames of imagery were collected with each of the four cameras, totaling over 60,000 frames of data. This was accomplished with 148 flight lines and approximately 3,110 flight line kilometers, covering an area of approximately 650 $\mathrm{km}^{2}$.

Flight line management was handled by the Leica ALS-60 flight line management system (FMS). Mission profiles created by technicians at Kucera International were uploaded to the system before every flight as new target areas were identified on a daily basis by the team centered in Rochester. The FMS is directly coupled to the lidar instrument and provides automatic sensor operation and tuning once the pilot has aligned the aircraft to a flight line. WASP is currently a completely decoupled and independent system from the FMS; this required the operator to manually begin and stop collection at the start and end of flight lines. Images from the WASP cameras were obtained every three seconds to provide ample overlap between frames and account for variations in ground speed and the occasional aircraft attitude change, due to high winds crossing from the mountains into the Port-au-Prince valley. At the end of each flight line, calibration imagery for the MWIR and LWIR sensors on WASP was acquired; calibration set points were adjusted to nominally bracket the energy observed in the scene (Arsenovic et al., 2009).

Weather remained a constant concern for successful collection missions. Early morning collection efforts were made for the outlying areas to minimize cloud shadows. The flight crew continued collection of imagery below the developing ceiling where possible to provide coverage to address the immediate need for reconnaissance information.

\section{Data Collection, Transfer, Processing, and Dissemination}

Up to 200 GB of raw imagery was collected every day, entering a workflow centered at RIT with the goal of disseminating ortho-rectified data products within 24 hours of raw data collection. Therefore, two very different transfer methods were used simultaneously: the data would be transferred to RIT over public networks and they would be shipped through a courier. This latter approach served as a contingency should the overnight network-based transfer fail. In practice, the shipped data were never required, since the overnight Internet transfers always completed before the next morning.

Once the imagery data arrived at UPRM, data were hosted on local equipment, from where it could be transferred to RIT in Rochester, New York. The primary network for this transfer was Internet2 (I2), a high speed network widely used by academic and research institutions. However, merely having access to I2 networks was insufficient to address the need for rapid data transfer. Nearly all file transfer tools are built on the Transmission Control Protocol (TCP) networking protocol, which was not designed for long distance networks with high latency (Consultative Committee for Space Data Systems, 1997). Thus, in this environment, the team could never achieve transfer speeds greater than $40 \mathrm{Mb} / \mathrm{s}$ from UPRM to RIT. This translated into over eleven hours to transfer a single collect of raw imagery and did not support the mission goals. However, a body of active research exists on the lossless transfer of data over an alternate protocol, namely User Datagram Protocol (UDP). UDP is a connectionless and lossy protocol, so it is not ideally suited for connection-oriented applications such as file transfer. For these reasons a number of applications for file transfer over UDP have appeared in recent years (Dickens and Gropp, 2002; He et al., 2002; Grossman and Gu, 2007). While tools like SABUL, FOBS, and GridFTP were considered for use, the application Tsunami was chosen for this effort based on prior experience.

The RIT Internet2 gateway was raised from 256 to 512 $\mathrm{Mb}$ per second for this effort, thereby matching the network gateway in use at UPRM. The tools were tuned on-the-fly for network performance (lag and packet loss) and by the end of the effort, peak data rates of $400 \mathrm{Mb} / \mathrm{s}$ could be achieved regularly, which is approximately ten times the traditional TCP data rate. With such drastically improved throughput, yielding average overall transfer rates of 200 to $300 \mathrm{Mb} / \mathrm{s}$, a full day's collection of raw data could be transferred to RIT in under two hours. This approach allowed the processing team to successfully support the "next-day-turnaround" goal of our processing workflow.

The WASP had relied on a post-processing workflow that utilized the ERDAS Photogrammetry Suite (LPS) to provide 


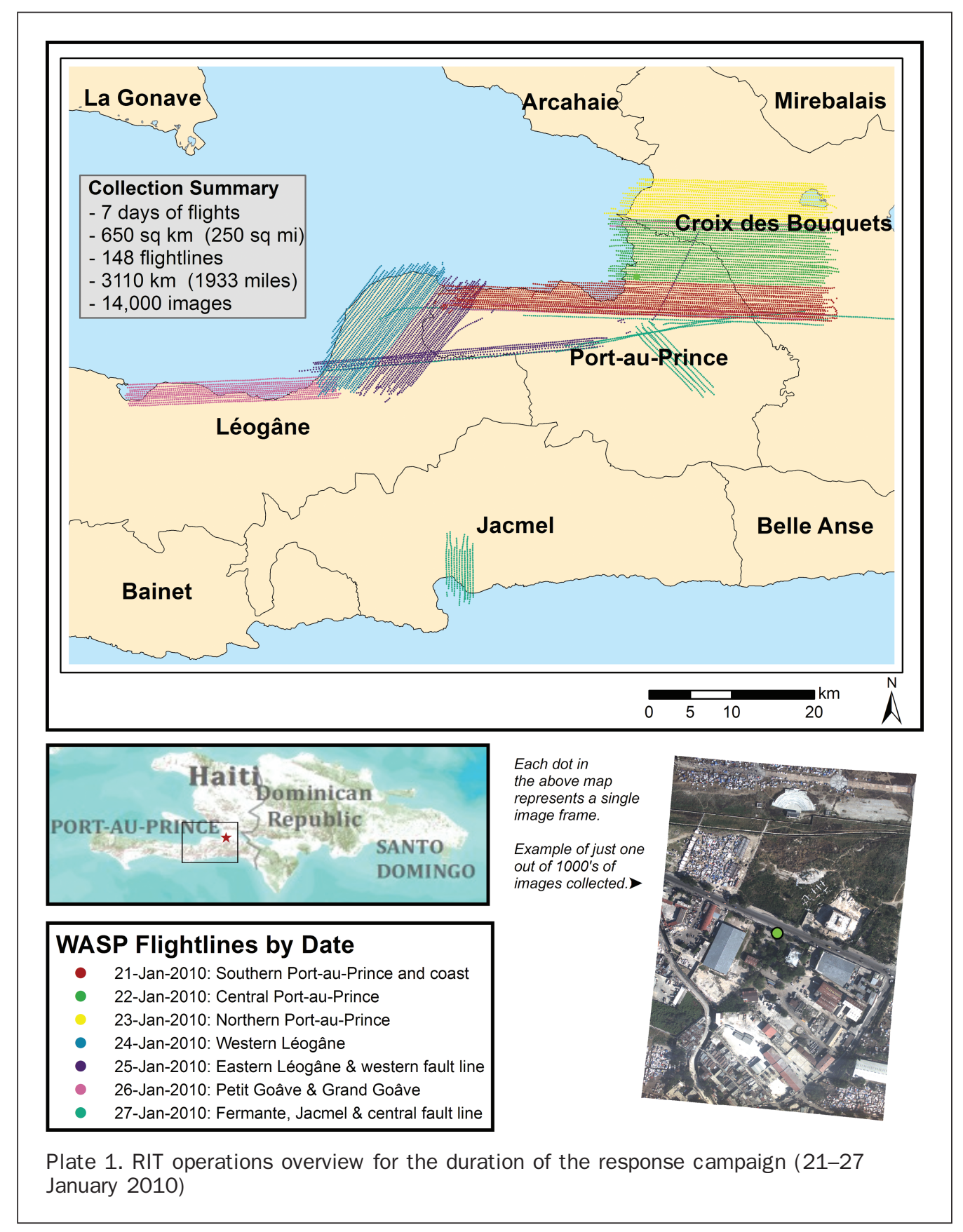

spatial resampling and ortho-rectification of collected imagery prior to the mission over Haiti. The LPS approach was utilized to deliver the ortho products from the first four Haiti mission days. It quickly became apparent that, given the massive number of frames to be processed, the work would have to be divided amongst multiple processing systems to achieve the quickest release of products possible. This process worked relatively well with a patchwork of Windows os workstations, although network congestion of the local file server quickly became a pacing issue. Raw image frames were originally ortho-rectified to 8- and 14-bit versions utilizing a digital elevation model (DEM) from the Shuttle Radar Topography Mission (SRTM) at $90 \mathrm{~m}$ spatial resolution and the data from the INS and Global Positioning System (INS/GPS). This enabled an immediate release of imagery shortly after each flight's raw data reached Rochester and before a more precise lidar-based DEM was created. Lidar data were processed to produce a $1 \mathrm{~m}$ DEM and surface height rasters, which were later used to re-process the data from WASP to increase the spatial accuracy of the ortho products to nominally $0.5 \mathrm{~m}$ root mean square error (RMSE) absolute position for the visible band camera. However, it became apparent upon WASP's return to Rochester that a new resampling engine and methodology would be necessary to speed product development and dissemination for the remainder of the mission data. The team had been working towards utilizing the open-source OSSIM tools (OSSIM, 2010) for WASP data processing prior to Haiti deployment. Production speed was substantially increased by finalizing the OSSIM-based workflow, since processing effectively could be distributed across multiple systems and scripted in a way that required minimal human interaction. 
The data products generated at RIT, namely the orthorectified imagery of both color and infra-red sensors, were combined with lidar based data products from Kucera (DEM and digital surface model (DSM) at varying resolutions) and distributed to various partners. These included ImageCat, the World Bank GFDRR group, Google ${ }^{\circledR}$, NGA, USGS, the University at Buffalo Virtual Disaster Viewer, and the Haiti Crisis Map Telascience site, among others. The bulk of the data dissemination was achieved over the Internet and Internet2 connections at RIT, although selected data sets were distributed using portable hard drives and overnight delivery services to alleviate network congestion. Approximately half a terabyte of products were generated per day during the effort. Eight terabytes of both intermediate and final data were generated in the RIT workflow, of which three terabytes were distributed for use by external agencies and organizations.

The first receivers of these data in many cases also agreed to host it for downloading by other partners. Although this approach had obvious logistical benefits, it quickly turned into an absolute necessity during the effort, since the number of other organizations that required copies of the data products produced at RIT far exceeded initial estimates. The team was thus able to meet download needs during the immediate response effort and in the months afterward by distributing the load across our collaborators. More than 130 terabytes had been downloaded directly from RIT alone by late-July 2011. Based on the success of this model, we recommend that the application of distributed file sharing services among organizations be evaluated in a future effort of this nature.

\section{Disaster Response Products}

This section discusses how a number of critical rapid turnaround disaster response products were generated based on the high spatial resolution imagery and the structural lidar data. Products included building damage assessments, location of internally displaced persons (IDPs), and hydrological products for assessment of the flood risk during the rainy season. It should be noted that the goal was rapid turnaround products at as high accuracy as possible without reliable ground truth data. The philosophy that disaster responders ". .. . would rather have best-guess disaster products available, as opposed to no information at all . .." permeated the response effort (Seth Spoelman, FEMA, personal communication). However, great care was taken in all instances to ensure that products were as reliable as possible and based on established research and algorithm development efforts.

\section{Post-Earthquake Damage Assessment}

Three key international organizations (the World Bank, the United Nation Institute for Training and Research's (UNITAR) Operational Satellite Applications Program (UNOSAT), and the European Commission's Joint Research Centre (JRC)) collaborated to produce a rapid assessment of building damage in Haiti using very high-resolution aerial imagery. This collaboration falls within the framework of the joint declaration signed in 2008 between the World Bank, the European Commission, and the United Nations Development Group on post-crisis Assessments and Recovery Planning (United Nations, 2008). It is clear from the Haiti experience that the future of post-disaster damage assessments has changed dramatically and that the "bar has been raised" for the next event.

The main outcome from the joint collaboration was a damage assessment, based on the use of high $(\sim 2 \mathrm{~m})$ and very high $(0.15 \mathrm{~m})$ spatial resolution multispectral imagery.
Damage was measured in terms of the number of buildings in different damage states. The joint team agreed to use a damage scale that was developed several years ago in Europe, the European Macroseismic Scale 1998 (EMS-98) version (Grünthal et al., 1998; Schwarz et al., 2006; Musson et al., 2010), to ensure standardization of the resultant damage assessment product. The EMS-98 scale includes five damage grades: 1 - no visible damage; 2 - minor damage; 3 moderate damage; 4 - very heavy damage; and 5 - destroyed (Grünthal et al., 1998). Fundamentally, this was one of the most important decisions made by the World Bank-UNOSATJRC team, because it ensured a common basis for measuring the effects of the earthquake. By agreeing to use this scale, the damage assessment results from each organization could be combined without significant re-adjustment.

Table 2 shows the damage totals for the ten communes that were significantly affected by the earthquake. In total, over 90,000 buildings were either destroyed (level 5) or heavily damaged (level 4) based on an assessment of the post-earthquake imagery. This represents slightly less than one third of the building inventory in the affected areas. Most of the damage occurred in Port-au-Prince; however, significant numbers of buildings were also destroyed in Carrefour, Delmas, Leogane, and Petion-Ville. This damage translates roughly to over 26 million $\mathrm{m}^{2}$ in building area affected, based on median floor area estimates for different occupancy uses, with approximately one-third of this total associated with buildings that require significant repairs or replacement. The total repair cost to buildings is estimated to be well over $\$ 6$ billion USD (EC-JRC, 2010).

\section{Crowd-sourcing}

Crowd-sourcing or human computation is a relatively new development and certainly a new concept in the area of disaster response. The notion of using a large community of experts to help perform damage assessments in disasters has been introduced by a number of individuals and organizations (Coyle and Meier, 2009). With the Internet becoming the foundation of "social networking," it is not unexpected that virtual damage surveys using high-resolution imagery displayed on Google Earth-type platforms emerged as a major source of damage information for the Haiti PDNA. After the Haiti earthquake, hundreds of individuals from around the globe actively sought ways of contributing their expertise, time, and knowledge to help support the people of Haiti.

ImageCat, Inc. working with a network of partners including the Earthquake Engineering Research Institute

TABle 2. Number of Buildings Grouped by the ems-98 DAMAge GRADE AND COMMUNE

\begin{tabular}{lrrrrr}
\hline & \multicolumn{5}{c}{ EMS-98 Damage Grades } \\
\cline { 2 - 6 } COMMUNE & \multicolumn{1}{c}{5} & \multicolumn{1}{c}{4} & \multicolumn{1}{c}{3} & \multicolumn{1}{c}{2} & \multicolumn{1}{c}{1} \\
\hline Carrefour & 2763 & 5905 & 5920 & 3220 & 35219 \\
Cite Soleil & 1012 & 549 & 1073 & 576 & 6403 \\
Delmas & 5012 & 2814 & 5064 & 2881 & 29478 \\
Grand-Goave & 148 & 541 & 421 & 276 & 2175 \\
Gressier & 565 & 289 & 567 & 319 & 3436 \\
Jacmel & 214 & 1785 & 1489 & 857 & 8799 \\
Leogane & 2220 & 5985 & 4139 & 2360 & 24736 \\
Petion-Ville & 2027 & 906 & 1693 & 708 & 10614 \\
Petit-Goave & 173 & 104 & 167 & 116 & 770 \\
Port-Au-Prince & 9902 & 15257 & 12351 & 6699 & 62693 \\
Tabarre & 532 & 365 & 663 & 383 & 3914 \\
Total & $\mathbf{2 4 0 6 2}$ & $\mathbf{3 4 5 0 0}$ & $\mathbf{3 3 5 4 6}$ & $\mathbf{1 8 3 9 5}$ & $\mathbf{1 8 8 2 3 6}$ \\
\hline
\end{tabular}


(EERI), reached out to the engineering and scientific community to help in quantifying the extent of damage caused by the Haiti earthquake. EERI is a professional organization of engineers and scientists that are dedicated to reducing the effects of earthquake on people and structures. Founded in the late 1960's, its membership exceeds several thousand individuals (Earthquake Engineering Research Institute, 2010). In direct response to this event, ImageCat and EERI formed the GEO-CAN (Global Earth Observation - Catastrophe Assessment Network) community to help the World Bank in its effort to quantify building damage. The GEO-CAN community consisted of over 600 experts from 23 different countries, with over 60 major universities, 20 government agencies and non-profit organizations, and over 50 private companies represented (Virtual Disaster Viewer, 2010). Using very high-resolution aerial imagery from RIT and Google, this community was able to identify the number of heavily-damaged and destroyed buildings in greater Port-auPrince. A major product from this effort was the delineation of the pre-earthquake footprints of these buildings that would later be used by the joint WB-UNOSAT-JRC effort to quantify the amount of building area that needed to be replaced or repaired. In addition, the footprints offered an efficient way of combining the damage assessment results from the World Bank effort (GEO-CAN) and the UNOSAT and JRC teams. Using crowd-sourcing as the main information technology tool for post-disaster damage assessment, the GEO-CAN community was able to deliver its first count of damaged buildings in less than a week. Efforts by the World Bank, EERI, and ImageCat are ongoing to institutionalize the GEO-CAN community as a permanent tool in the World Bank damage assessment toolbox.

\section{Location of Displaced Persons}

Another important information element to the relief effort was the location of Internally Displaced Persons (IDPs), necessary because damaged structures forced a large number of residents to flee their homes. Questions arose as to the location and dynamics of refugee camps, e.g., assessing the growth of informal settlements and routes to reach these locations. Dynamic analysis of camps was not possible, since this effort did not produce multi-temporal coverage of a single area. However, it was possible to derive the location of camps over a range of sizes through detection of an indicative signature: blue tarps. It is not known whether tarps were provided by relief organizations or were simply the material of choice, but it quickly became apparent that there was a proliferation of bright blue tarps that were being used for shelter by IDPs. It was also observed that there were few other blue objects in the imagery, thereby rendering this shelter material relatively unique. Researchers at RIT decided to automatically identify and locate blue objects in the imagery through the use of multispectral image processing techniques.

It was demonstrated that the blue tarps could be located with a relatively simple Mahalanobis distance classifier (Schott, 2007). The classifier was trained by collecting known blue tarp pixels across a number of individual frames of imagery. The mean RGB values from the samples were computed and the same collection of pixels was used to estimate the covariance in the three-band signature. These target mean signature and covariance matrices were then used to test each pixel in terms of its likelihood of being a blue tarp. Detections were declared above a threshold to provide a confidence level. The algorithm was implemented in the IDL/ENVI (version 4.6) software package and was used to automatically analyze over a thousand frames of imagery from the collection (Messinger et al., 2010).

A key component of any detection methodology is the presentation of the results. Here, where the likely consumers of the information were first responders and not remote sensing scientists, it was decided to output the results using a common geospatial information platform, namely Google Earth. The algorithm utilized the geo-information in the imagery to produce KML files showing the locations of the detected blue tarps. Clusters of detections, which were anticipated due to the high spatial resolution of the imagery and a priori knowledge of the size of the targets, were identified to avoid "clutter" in the output detection map and located with push pins in the KML files. Example results from the detection of IDPS are shown in Plate 2 for the area of Jacmel, along the southern coast of Haiti. An added benefit to this detection methodology and visualization scheme is that the final information product requires relatively low transmission bandwidth; each KML file contains only the $\mathrm{x}, \mathrm{y}$ coordinates of the push pins and therefore can be very small in size.

\section{Hydrological Modeling}

The $1 \mathrm{~m}$ DEM, based on the high-resolution lidar data, enabled the application of terrain and hydrologic flow analysis algorithms that delineate the current drainage patterns and watersheds (Renschler, 2003). Renschler (2006) illustrated that even localized topographic fluctuations in dynamic topographic landscapes, such as volcanoes, can potentially cause drastic drainage pattern changes that result in significant alterations to hydrologic regimes and surface water runoff dynamics. Such analyses become important when assessing the location and relocation of temporary shelters to avoid potential conflicts, due to flooding and landslides caused by the storm events during the rainy season in Haiti (May through November). Frequently flooded, open, and flat urban areas along larger drainages that were not used as permanent residential areas were likely used as temporary shelter immediately after the disaster. Since most of the urban storm water drainages follow existing channels and roads, debris of collapsed buildings can cause flow changes and flooding in locations previously thought safe. Plate 3 illustrates regional and local drainage patterns based on two different critical source areas (the drainages are delineated when the watershed size is larger than 1 ha or $1 \mathrm{~km}^{2}$, respectively). It is obvious that relocated debris piles, as well as mobile debris and mudslides during floods, could cause additional blockages of bridges and roadways that are not represented in the collected data sets.

\section{Conclusions}

The ImageCat-RIT-Kucera International airborne response following the 2010 Haiti earthquake has demonstrated the usefulness and potential implementation of an end-to-end collection, transfer, processing, and product development chain. Multi-modal remote sensing data sets, including visible, thermal infrared, and lidar data, enabled the development of a variety of disaster response products. These included building damage assessments by the GEO-CAN community for use by the World Bank reconstruction effort, headed by ImageCat and verified using crowd-sourcing approaches, 3D lidar-based analysis of the fault line by the USGS, target detection of displaced persons towards directing relief efforts, and hydrological modeling for determining flood and mud slide risks.

The unique components of this effort included the multi-modal data types (i.e., visible/thermal infrared imagery and lidar), rapid deployment, transfer of data using Internet2 networks at rates that approached $400 \mathrm{Mb} / \mathrm{s}$ for next day processing and delivery, public availability of the data, and coupled disaster product development. Finally, the entire effort was continuously guided by user needs and feedback, 


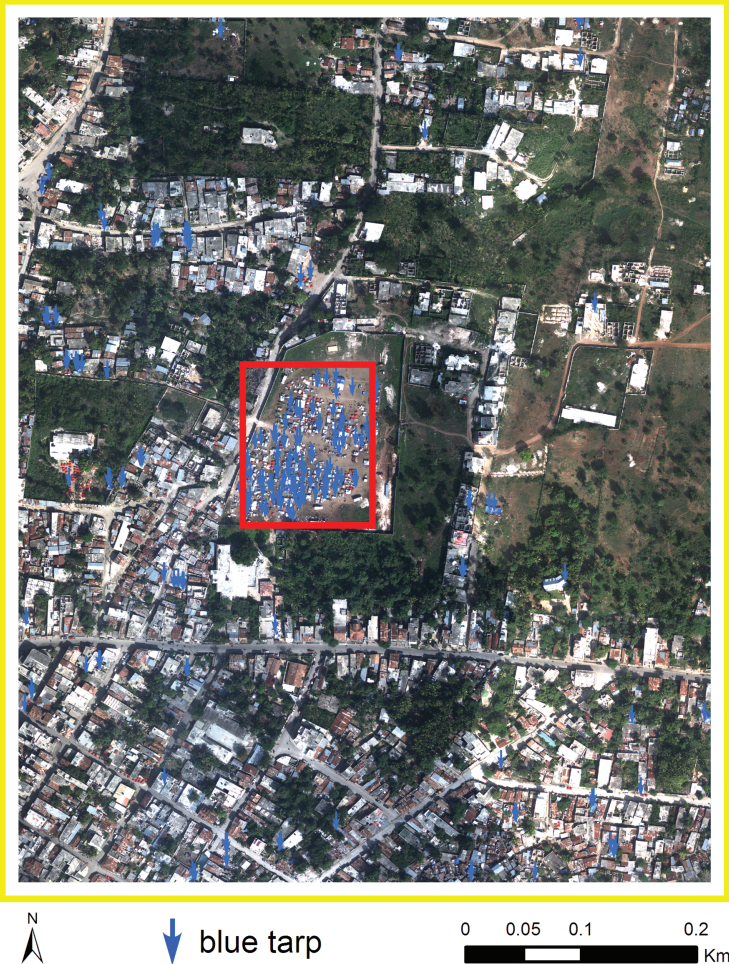

(a)

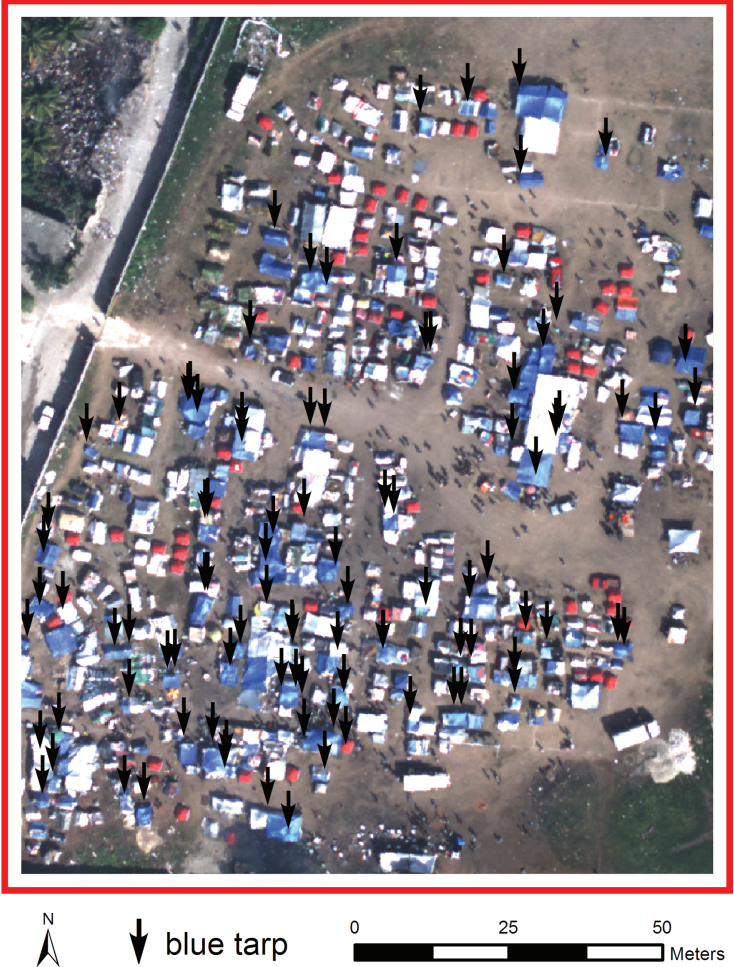

(b)

Plate 2. KML file output for the blue tarp, Mahalanobis distance-based, target detection algorithm. Note the (a) generalized representation at small scales, and (b) detailed locations as the user zooms in to larger scales (arrows indicate actual detections on WASP imagery).

e.g., World Bank and USGS coverage and product needs for building damage assessment and fault line lidar data, respectively. This complete workflow arguably contributed to a new phase for approaching geospatial disaster response and management: responder-driven specification of data types and products, with novel approaches to transfer, processing, and product development for rapid disaster response. However, care should be taken during future disaster events to constrain data and products to those needed by the immediate responders and decision makers at ground level. Hence the philosophy of a continuous and open feedback loop between engineer (system developer), scientist (algorithm developer), and end-user (application specialist) is essential to effective disaster response.

While the area of remotely-sensed damage assessment and other diasaster product development took a quantum leap forward during the international reponse to the Haiti earthquake, there is still significant room for improvement. Some of the areas that deserve further investigation or review are:

1. The establishment of standard operating procedures to ensure that damage assessments performed by multiple groups are consistent, transparent, and fully compatible. An important element of any protocol will be standard definitions of damage and a damage scale based primarily on remotely-sensed imagery. Current efforts by the joint World Bank-UNOSAT-JRC team are focusing on a preliminary set of guidelines in this area (Eguchi et al., 2010).

2. The availability of high quality imagery in near real-time: a key factor in the success of the Haiti response was having access to very high-resolution imagery within a matter of days. Rapid access to these data allowed a number of operations to proceed with greater confidence, i.e., deployment of emergency personnel to assess the safety of damaged structures. The fear of "overcommitting" resources before the response priorities were known, was somewhat mitigated by having these early "snapshots" of the devastation caused by the earthquake.

3. The value and exploitation of multi-modal data sets should receive recognition and focus. It was evident that different modalities contributed to distinct disaster response products and needs. High spatial resolution airborne imagery allowed the GEO-CAN team to provide reliable building damage assessments, while lidar data enabled the development of structural data products. We suggest that continued research and development focus on semi-automated approaches to quantify damage, debris, road trafficability, and detecting targets of interest.

Only when disaster responders can dictate which geospatial products should be delivered at what rates, can we truly address the urgent need for specific response tools. Finally, the collaboration across groups, agencies, and nations is essential to saving lives and aiding in recovery efforts. The manner in which various groups interacted during the Haiti response, shared data and knowledge, and responded to product needs, can only contribute to an improved response model when inevitable natural diasters strike again. This inter-agency collaboration is equaled in importance only by standing up a rapid response team that complement each other in terms of addressing various facets in the disaster reponse chain. This was key to the effective remote sensing or geospatial response in Haiti, or put differently, any successful response effort requires a team 


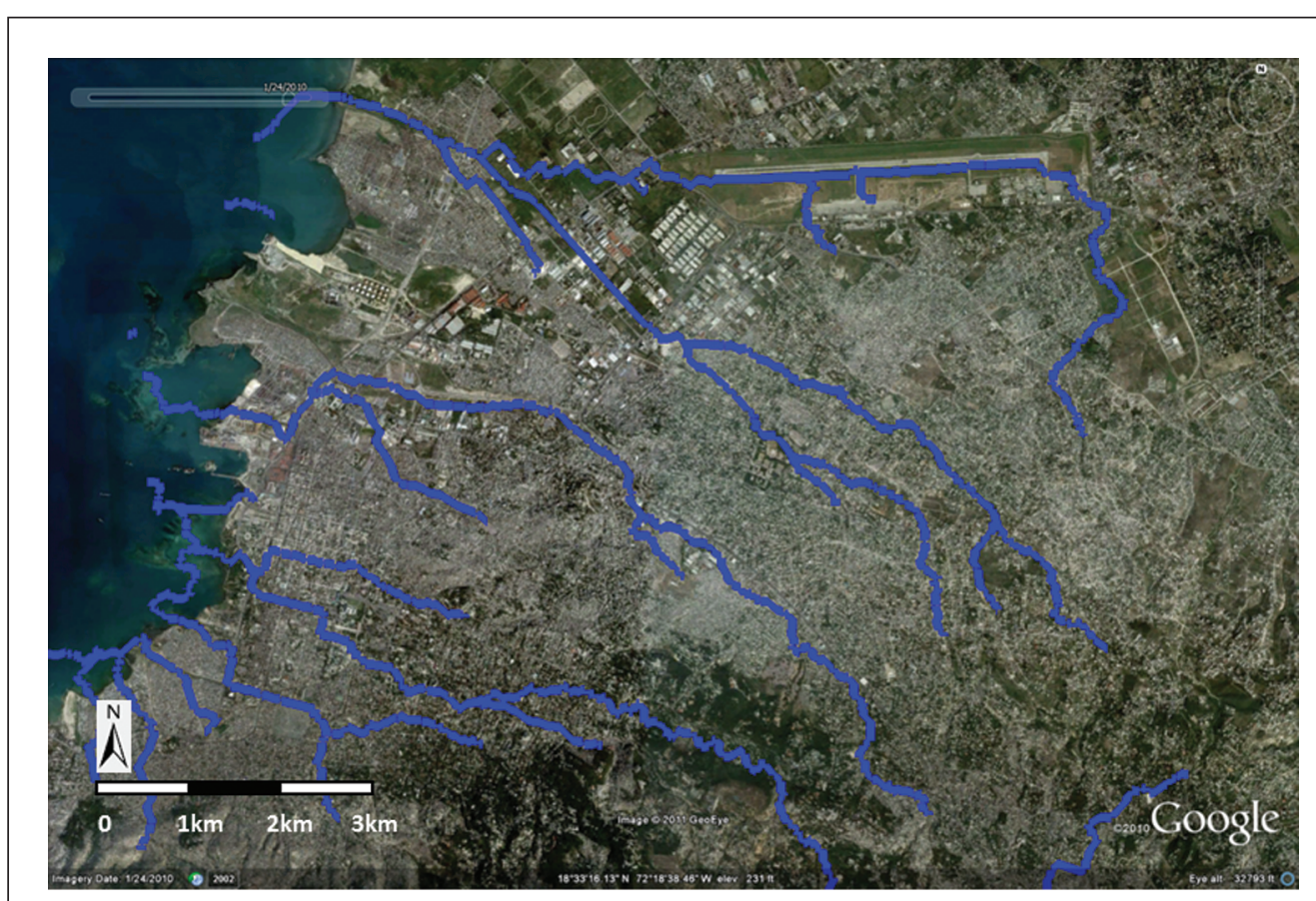

(a)

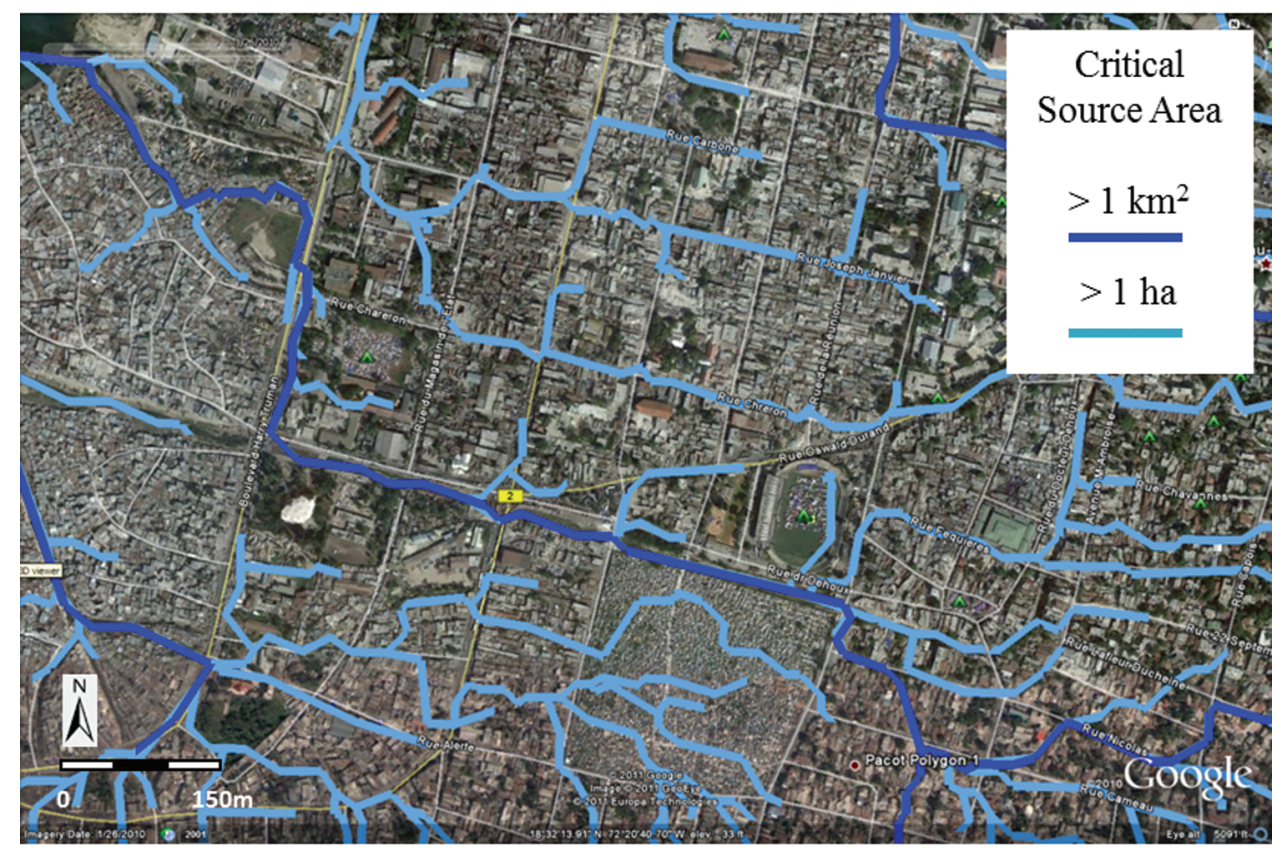

(b)

Plate 3. Drainage patterns of critical source areas at (a) regional ( $>1 \mathrm{~km}^{2}$ only), and (b) local scales. Drainages follow mainly roads, but may not follow built ditches (Images courtesy of Google, Inc.).

with individual members that contribute to a better understanding of technology, how to implement the technology, and what should be delivered by the technology.

\section{Acknowledgments}

The ImageCat-RIT-Kucera International team gratefully acknowledges the financial support from the World Bank GFDRR group. We furthermore acknowledge the NSF Partner- ships for Innovation Program (NSF Award No. 0917839) for the funding that made the IPLER project possible; IPLER directly led to the collaboration that enabled the disaster response effort. We want to thank Michael Aslaksen (NOAA), Brenda Jones (USGS), Colonel Byron Mathewson (AFSOUTH), and Seth Spoelman (FEMA) for logistical support and mission advice. Southern Command personnel, particularly those with Air Force Southern (12 ${ }^{\text {th }}$ Air Force), exhibited the highest standards of professionalism and were extremely helpful. 


\section{References}

Arsenovic, M., C. Salvaggio, A. Garrett, B. Bartlett, J. Faulring, R. Kremens, and P. Salvaggio, 2009. Use of remote sensing data to enhance the performance of a hydrodynamic simulation of a partially frozen power plant use of remote sensing data to enhance the performance of a hydrodynamic simulation of a partially frozen power plant cooling lake, Proceedings of Thermosense XXXI (D. Burleigh and R. Dinwiddie, editors), SPIE, Vol. 7299.

Consultative Committee for Space Data Systems, 1997. Space Communication Protocol Specification (SCPS) Rationale, Requirements, and Application Notes, CCSDS-710.0-G-0.3, National Aeronautics and Space Administration (US), April, p. 2-2.

Coyle, D., and P. Meier, 2009. New Technologies in Emergencies and Conflicts: The Role of Information and Social Networks, Washington, D.C. and London, UK: United Nations FoundationVodafone Foundation Partnership, $60 \mathrm{p}$.

Dickens, P.M., and W. Gropp, 2002. An evaluation of object-based data transfers over high performance networks, Proceedings of the $11^{\text {th }}$ IEEE International Symposium on High Performance Distributed Computing, 22-26 July, Edinburgh, Scotland, 10 p.

Earthquake Engineering Research Institute, 2010. URL: http://www.eeri.org (last date accessed: 15 June 2011).

Eguchi, R.T, S.P. Gill, S. Ghosh, W. Svekla, B.J. Adams, G. Evans, J. Toro, K. Saito, and R. Spence, 2010. The January 12, 2010 Haiti earthquake: A comprehensive damage assessment using very high resolution aerial imagery, Proceedings of the $8^{\text {th }}$ International Workshop on Remote Sensing for Disaster Management, Tokyo, Japan, 30 September-01 October 2010, 8 p.

EC-JRC (European Commission Joint Research Centre, United Nations Institute for Training and Research - Operational Satellite Applications Programme, World Bank, and the Centre National d'Information Geo-Spatial), 2010, Building Damage Assessment Report - Post Disaster Needs Assessment and Recovery Framework, version 3.0, 11 March 2010.

Grossman, R., and Y. Gu, 2007. UDT: UDP-based data transfer for high-speed wide area networks, Computer Networks, 51(7):1777-1799.

Grünthal, G. (editor), R.M.W. Musson, J. Schwarz, and M. Stucchi, 1998. European Macroseismic Scale 1998 (EMS-98), Cahiers du Centre Européen de Géodynamique et de Séismologie 15, Centre Européen de Géodynamique et de Séismologie, Luxembourg, $99 \mathrm{p}$.

He, E., J. Leigh, O. Yu, and T.A. DeFanti, 2002. Reliable blast UDP: Predictable high performance bulk data transfer, Proceedings of the IEEE International Conference on Cluster Computing, 23-26 September, Chicago, Illinois, p. 317-324.
Li, Y., A. Vodacek, R.L. Kremens, and A. Ononye, 2005. A hybrid contextual approach to wildland fire detection using multispectral imagery, IEEE Transactions on Geoscience and Remote Sensing, 43:2115-2126.

Li, Y., A. Vodacek, and Y. Zhu, 2007. An automatic statistical segmentation algorithm for extraction of fire and smoke regions, Remote Sensing of Environment, 108:171-178.

McKeown, D., J. Cockburn, J.W. Faulring, R.L. Kremens, D. Morse, H.E. Rhody, and M. Richmond, 2004. Wildfire Airborne Sensor Program (WASP): A new wildland fire detection and mapping system, Proceedings of the US Forest Service Remote Sensing Applications Conference, Salt Lake City, Utah, 05-09 April.

Messinger, D.W., J.A.N. van Aardt, D. McKeown, M. Casterline, J. Faulring, N. Raqueño, B. Basener, and M. Velez-Reyes, 2010. High resolution and LiDAR imaging support to the Haiti earthquake relief effort, Proceedings of SPIE Imaging Spectrometry (S. Shen and P. Lewis, editors), 01-05 August, San Diego, California, Vol. 7812, 12 p.

Musson, R.M.W., G. Grünthal, and M. Stucchi, 2010. The comparison of macroseismic intensity scales, Journal of Seismology, 14(2):413-428.

Ononye, A.E., A. Vodacek, and E. Saber, 2007. Automated extraction of fire line parameters from multispectral infrared images, Remote Sensing of Environment, 108:179-188.

OSSIM, 2010. Awesome image processing, URL: http://www. ossim.org/OSSIM/OSSIM_Home.html (last date accessed: 15 June 2011).

Renschler, C.S., 2003. Designing geo-spatial interfaces to scale process models: The GeoWEPP approach, Hydrological Processes, 17:1005-1017.

Renschler, C.S., 2005. Scales and uncertainties in volcano hazard prediction - Optimizing the use of GIS and models, Journal of Volcanology and Geothermal Research, 139(1-2):73-87.

Schott, J.R., 2007. Remote Sensing: The Image Chain Approach, Oxford University Press, New York, 666 p.

Schwarz, J., M. Raschke, and H. Maiwald, 2006. Comparative seismic risk studies for German earthquake regions on the basis of the European macroseismic scale EMS-98, Natural Hazards, 38(1-2):259-282.

United Nations, 2008. Joint Declaration on Post-Crisis Assessments and Recovery Planning, URL: http://www.undg.org/docs/9419/ trilateral-JD-on-post-crisis-assessments-final.pdf (last date accessed: 15 June 2011).

Virtual Disaster Viewer, 2010. URL: http://www.virtualdisasterviewer. com (last date accessed: 15 June 2011).

World Bank, 2010, World Bank response to Haiti earthquake, URL: www.worldbank.org/haiti earthquake (last date accessed: 15 June 2011). 